ESJ Natural/Life/Medical Sciences

\title{
Identificación De Liderazgo Académico Para El Emprendimiento De Enfermería En La Región De Orizaba, Veracruz, México
}

\author{
Abril Camarillo Quiroz \\ Doctorante del colegio Interdisciplinario de Especialización, docente de la \\ Facultad de Enfermería \\ Leticia Méndez Jiménez \\ Docente de la Facultad de Enfermería de Orizaba Veracruz \\ Gloria Zita Roa Tostado \\ Docente de la Facultad de Enfermería de Orizaba Veracruz \\ Dr. Manuel González-Pérez \\ Docente del Colegio Interdisciplinario de Especialización
}

Doi:10.19044/esj.2021.v17n3p284

Submitted: 18 August 2020

Accepted: 25 January 2020

Published: 31 January2021
Copyright 2021 Author(s)

Under Creative Commons BY-NC-ND

4.0 OPEN ACCESS

Cite As:

Camarillo Quiroz A., Méndez Jiménez M., Zita Roa Tostado G. \& González-Pérez M. (2021). Identificación De Liderazgo Académico Para El Emprendimiento De Enfermería En La Región De Orizaba, Veracruz, México. European Scientific Journal, ESJ, 17(3), 284.

https://doi.org/10.19044/esj.2021.v17n3p284

\section{Resumen}

El desarrollo de proyectos de emprendimiento no es una opción de ingreso económico para profesionales de enfermería, no se observa liderazgo académico que influya en la creación de negocios en el cuidado de la salud. El objetivo de este estudio fue identificar la existencia de liderazgo académico en profesionales de enfermería para el emprendimiento. Se realizó una investigación documental de los programas de estudio de 5 universidades en México, posteriormente se aplicó un instrumento de recolección de datos con respuestas dicotómicas a 70 profesionales de enfermería egresados de diferentes universidades del país. Los programas de estudios revisados no cuentan con materias relacionadas con la creación de negocios en salud, los académicos que imparten clases en las universidades no cuentan con experiencia en creación de negocios en enfermería, los egresados de enfermería no emprenden negocios para el cuidado de la vida y salud de las personas, familias o comunidades, por lo tanto, no son gestores de su propia fuente laboral. Como conclusión se 
obtiene que no existe liderazgo académico para el emprendimiento en enfermería, los egresados buscan emplearse en instituciones gubernamentales o privadas de salud a pesar de las problemáticas de empleabilidad a las que se enfrentan. Se considera esencial que las universidades integren a sus programas de estudios materias que orienten a los egresados a emprender negocios para el cuidado de la salud de las personas.

Palabras clave: Liderazgo Académico, Enfermería, Cuidado, Emprendimiento

\title{
Academic Leadership In Nursing Entrepreneurship In Mexico
}

\author{
Abril Camarillo Quiroz \\ Doctorante del colegio Interdisciplinario de Especialización, docente de la \\ Facultad de Enfermería \\ Leticia Méndez Jiménez \\ Docente de la Facultad de Enfermería de Orizaba Veracruz \\ Gloria Zita Roa Tostado \\ Docente de la Facultad de Enfermería de Orizaba Veracruz \\ Dr. Manuel González-Pérez \\ Docente del Colegio Interdisciplinario de Especialización
}

\begin{abstract}
The development of entrepreneurship projects is not an economic income option for nursing professionals, academic leadership is not observed, which influences the creation of businesses in health care. The objective of this study was to identify the existence of academic leadership in nursing professionals for entrepreneurship. A documentary investigation of the study programs of 5 universities in Mexico was carried out, subsequently a data collection instrument with dichotomous responses was applied to 70 nursing professionals graduated from different universities in the country. The study programs reviewed do not have subjects related to the creation of health businesses, the academics who teach at the universities do not have experience in the creation of businesses in nursing, the nursing graduates do not undertake business for life care and health of individuals, families or communities, therefore they are not managers of their own source of employment. Conclusions it is obtained that there is no academic leadership for entrepreneurship in nursing, graduates seek employment in government or private health institutions despite the employability problems they face. It is
\end{abstract}


considered essential that universities integrate into their study programs subjects that guide graduates to undertake businesses for the care of people's health.

Keyword: Academic Leadership, Nursing, Care, Entrepreneurship

\section{Introducción}

La práctica de enfermería se encuentra fundamentada en el Proceso Enfermero, que es el método para brindar cuidados profesionales a las personas, familias y comunidades, comprende las etapas de valoración, diagnóstico, planificación, ejecución y evaluación, obteniendo resultados en la salud de las personas, familias y comunidades que atiende.

Los egresados de enfermería se enfrentan a diferentes problemáticas de desempleo, algunos de ellos inician a laborar, en ámbitos hospitalarios privados o en programas gubernamentales temporales percibiendo salarios mínimos, sin generar derechos básicos de prestaciones, otros profesionales realizan jornadas gratuitas con la promesa de ser contratados en un futuro y otro grupo de profesionales emigran de su lugar de origen o terminan empleándose en otras actividades distintas a su profesión.

Estas experiencias demuestran que los profesionales de enfermería visualizan el empleo como la única opción para generar recurso financiero en comparación con otros profesionales que eligen la apertura de consultorios, despachos o asociaciones independientes o con otros colegas y posicionar sus servicios en el mercado laboral.

Los profesionales de enfermería carecen de formación académica que les permita visualizar que, al egresar del ámbito universitario, pueden establecer negocios para el cuidado de la salud de las personas, realizando funciones asistenciales de promoción para la salud, prevención de enfermedades, intervención para el cumplimiento de un tratamiento específico de curación y/o rehabilitación.

Los programas de estudio de las universidades que ofertan la carrera de licenciatura en enfermería no contemplan materias que les permita a los futuros egresados visualizar el cuidado de la salud como una opción atractiva de oferta y demanda en el mercado y generar su propia fuente de ingreso económico.

El emprendimiento hoy en día se ha convertido en un conocimiento básico de enseñanza en las universidades, pues desempeña un papel importante para el desarrollo económico de los países, por la incubación de proyectos productivos de innovación y tecnología en las diferentes profesiones. Las universidades que imparten la carrera de licenciatura de enfermería requieren implementar estos conocimientos en sus programas de estudio de enfermería, permitiendo a sus egresados, adquirir competencias emprendedoras y desarrollar proyectos del cuidado de la vida y la salud de las personas, con el objetivo de crear una cultura de prevención de enfermedades, identificación 
oportuna de riesgos para la pérdida de la salud, prácticas terapéuticas del bienestar etc.

\section{Desarrollo}

\section{Funciones de enfermería}

El cuidado de enfermería es una actividad cuyo valor personal y profesional está dirigido a la conservación, restablecimiento y autocuidado de la vida y se fundamenta en la relación terapéutica del profesional de enfermería - paciente. El cuidado profesional de las personas incluye teoría y método científico que tienen por finalidad principal mantener a los seres humanos vivos y sanos con el propósito de perpetuar la especie, por lo tanto el cuidado es mantener la vida, asegurando la satisfacción de un conjunto de necesidades para la persona, que en continua interacción con su entorno, vive experiencias de salud (Colliere, 2003, p.113).

Desde un punto de vista administrativo, los servicios profesionales de enfermería se refieren a la preservación de la vida humana, la promoción y restauración de la salud, la ejecución de acciones derivadas del diagnóstico y tratamiento médico y el deber de velar por la mejor administración de los recursos de asistencia para el sujeto de cuidado, cuando éste solicita atención en las instituciones de salud (Hernández, 2020, p 3).

La atención de enfermería actualmente se observa un enfoque especial en intervenciones curativas dentro de las instituciones de salud, no considerando los aspectos de promoción para la salud, prevención de enfermedades y la rehabilitación de la salud como una oportunidad para emprender negocios en el cuidado de las personas y obtener recursos económicos.

Las funciones sustantivas del quehacer de la enfermería profesional son: asistenciales, educativas, administrativas y de investigación.

Las funciones asistenciales comprenden intervenciones en la atención del cuidado de las personas sanas o enfermas, implica el cuidado mismo de la vida y la salud para la permanencia y trascendencia del ser humano (Santa Isaza 2020 p.20).

Las funciones educativas de enfermería contemplan las intervenciones de enseñanza en salud que se brindan a las personas, al personal en formación y/o a otros profesionales (García y Gallegos-Torres 2020 pp. 272-273).

Las funciones administrativas, se refieren al papel principal de un jefe, gerente o gestor en enfermería y consiste en planificar, organizar, dirigir y controlar los recursos financieros, humanos y materiales, bajo el propósito de cumplir eficazmente los objetivos de la institución (Santa Isaza 2020 p.22).

Finalmente la función de investigación resalta a enfermería como una ciencia y arte del cuidado humano, donde se requiere una revisión continua de estrategias, técnicas y procedimientos, validación de sus teorías y la generación 
de nuevos conocimientos, que solo es posible, a través de la investigación para mejorar la praxis (Benítez 2020 p.1).

El denominado reto Nightingale Challenge 2020 que propone el Congreso del Consejo Internacional de Enfermeras, tiene como propósito ayudar a desarrollar la próxima generación de enfermeras y enfermeros jóvenes como líderes, profesionales y defensores de la salud, y demostrar que la enfermería es una profesión emocionante y gratificante (Bermúdez 2020 p.1).

Como se observa se puede argumentar que los profesionales de enfermería son responsables de cuidar la salud de la población y generar proyectos dentro y fuera de las instituciones públicas y privadas que impacten en la conservación de una vida sana, la disminución de recursos económicos para las personas y para las instituciones públicas de salud y un aumento en la demanda de servicios profesionales de enfermería privada.

Según Pedroza-Zapata y Silva-Flores (2020 p.1) las universidades actúan como un factor importante para el desarrollo económico y tecnológico de un país mediante su papel sustantivo de educación y es en las universidades donde se adquieren las competencias valiosas para el proceso de innovación y emprendimiento de las empresas e industrias.

En la disciplina de enfermería se observa que los programas de estudio continúan en su mayoría enfocados a obtener competencias disciplinares del cuidado centradas en la atención curativa, sin integrar innovación en los conocimientos que le permitan al egresado, explorar las diferentes alternativas del cuidado y solucionar problemas durante los diferentes procesos de la vida humana otorgando un cuidado integral.

Según Navarro y Pacheco (2020 p.3) no es aceptable continuar con las estructuras y prácticas educativas tradicionales pasadas, si se desea innovar en los centros educativos, se debe partir de identificar problemáticas y crear alternativas nuevas, las innovaciones educativas se comprenden en el sentido de un cambio, ya sea de paradigma, hacia la inclusión o hacia el desarrollo de la competencia social y ciudadana.

Los desafíos para el desarrollo a nivel país, ha situado la atención en el sistema educativo como parte de las soluciones a las tensiones sociales, políticas y económicas existentes y para resolver las problemáticas en salud se requiere de profesionales altamente competitivos que generen ideas innovadoras que permitan brindar alternativas de solución a las diferentes problemáticas (Acosta y Leyva 2020 pp. 14-15).

Los profesionales de enfermería son responsables de brindar cuidados en los diferentes procesos vitales del ser humano tales como: vigilancia del crecimiento y desarrollo en la vida intrauterina y extrauterina en todas sus etapas, ofrecer cuidados durante el proceso de una enfermedad, rehabilitación de las funciones físicas, mentales, emocionales y espirituales, así como el acompañamiento en procesos de dolor como en casos de pérdidas o muerte. 
La comprometida participación de la profesión de enfermería en la vida de las personas descubre un área de oportunidad para iniciar diferentes proyectos de emprendimiento en salud con posibilidades de crecimiento empresarial local, nacional e internacional, dirigiendo el cuidado de enfermería a ejercer una práctica privada ya sea individual o con otros profesionales de enfermería.

Las universidades tienen la responsabilidad social de impulsar a las diferentes disciplinas a incursionar en diferentes ámbitos organizacionales, para que al egresar de la universidad cuente con una amplia gama de posibilidades laborales que les permita incorporarse a una vida económicamente productiva de la manera más exitosa posible (Navarro y Pacheco 2020 p.4).

Las universidades que imparten la carrera de enfermería requieren innovar sus programas de estudio, integrando nuevas materias que traten de las diferentes prácticas terapéuticas, que permiten preservar una vida sana y no esperar a que las personas enfermen para recibir la atención de enfermería en instituciones de salud.

La práctica de enfermería moderna permite a los profesionales convertir el cuidado en un servicio de alta valía, donde las personas se encuentren dispuestas a intercambiar un bien monetario por un servicio de cuidado, impactando positivamente en la toma de decisiones de las personas con respecto a su salud, por lo que es fundamental que durante la formación, el alumno adquiera competencias de emprendimiento, mercadotecnia en salud, ofertas y demanda de un producto o servicio, reingeniería del cuidado, innovación, etc. que le permitan al futuro profesional gestionar su propio ámbito laboral.

Esta práctica de enfermería moderna daría como resultado la generación de fuentes de empleo, además de un importante ahorro económico en el consumo de medicamentos, servicios de hospitalización, y otros servicios terapéuticos y finalmente lo más importante una población con mejores condiciones de salud.

\section{Liderazgo académico}

Los maestros que imparten catedra a futuros profesionales de enfermería, desempeñan un papel imprescindible en la adquisición de competencias de cualquier disciplina, son ellos los que influyen en los alumnos para desarrollar la personalidad profesional. De acuerdo con Hernández y Romero (2020 p.5) el maestro es quien guía académicamente a lograr los objetivos en búsqueda del éxito de los alumnos, de ahí la importancia de que el docente se mantenga actualizado y adquiera nuevas competencias docentes que le permita ejercer un liderazgo transformacional.

Es recomendable que el profesor implique en su desempeño laboral competencias propias de liderazgo, que le permita propiciar aprendizajes significativos en sus alumnos y que los conduzcan a adquirir un pensamiento 
dinámico, orientado a la prevención y resolución de diferentes problemáticas de la sociedad, así como de conformar un pensamiento abierto al cambio y a la innovación, desarrollando un trabajo colaborativo que posibilite la consistencia, coherencia de las visiones, motivaciones y compromisos de sus integrantes (Gatica, Cruz, García, Vargas, Hernández y Tepal 2019 p.3).

El perfil profesional del docente está asociado a una imagen de docencia deseable y contextualizada que se convierte en un referente para quienes optan por la profesión, el rol docente viene enmarcado en un modelo sistémico e interdisciplinar, donde la docencia, la investigación, el saber, el saber hacer y querer hacer conformarán su acción formativa de los futuros egresados (Bozu y Herrera 2009 p.90 citado por Hernández y Romero 2020 p.4).

Según Guerrero y Conde (2020 p.2) comienza a vislumbrarse un proceso de innovación en la práctica docente en la pedagogía emprendedora en la cual se requiere de un docente preparado para asumir los cambios en los diferentes espacios de formación, adquirir nuevas competencias y habilidades pedagógicas para poder responder a los desafíos de una sociedad global, comprendiendo que los estudiantes de este tiempo se encuentran inmersos en la era digital con sistemas de información diversa y sofisticada, brindando un apoyo en la flexibilidad de pensamiento y capacidad para adaptarse a situaciones propias de un entorno cambiante.

Así mismo Gatica et al. (2019, p.2) expresan que el desarrollo de un liderazgo eficaz en el desempeño laboral de los docentes, se convierte en un punto crítico de trabajo para ser abordado por las instituciones de educación superior a partir de la comprensión de su propia realidad, ya que en una sociedad cada vez más exigente, se evidencia la imperiosa necesidad de asumir por parte del docente un rol de líder, que le permita transformar su quehacer cotidiano.

El perfil del docente de enfermería requiere estructurar una personalidad de liderazgo que contemple una vida ejemplar, con apertura de pensamiento para adquirir nuevos paradigmas que dirijan a la disciplina a desarrollar un espíritu emprendedor que le permita al profesional posesionarse en el mercado laboral.

\section{Emprendimiento en enfermería}

A finales del siglo XX, autores como Stevenson (1980) y Timmons (1998) desarrollaron investigaciones relacionadas con la mentalidad emprendedora y el modelo para emprender. Ambos autores consideran el emprendimiento como un sistema de gestión apoyado en oportunidades más que en los recursos, caracterizando en dos líneas de acción: a) los creadores de empresas que parten del diseño e implementación de un plan de negocios y b) los empleados como agentes activos en la innovación de procesos. (Vélez, Bustamante, Loor y Afcha, 2020 p.64). 
El emprendimiento se ha convertido en una de las bases transversales de la oferta académica superior, buscando formar jóvenes con visión emprendedora que sean agentes de transformación social local, nacional e internacional (Bayas, García, Chaguay y Sánchez 2020 p.3).

El emprendimiento es un término utilizado en la actualidad a nivel global, que busca fomentar en los individuos una visión diferente que les permita determinar oportunidades, no solo de crear nuevas empresas, sino también de ser proactivos e incorporar un mayor dinamismo a la solución de problemas o logro de objetivos personales y sociales (Bayas et al. 2020 p-4). El emprendimiento puede convertirse en una herramienta útil para que la profesión de enfermería inicie a incursionar en el ámbito empresarial del cuidado.

Las competencias son sistemas complejos de desempeño con idoneidad en un determinado contexto (Tobón 2006 p.1), para Fernández y Rey (2010 p.18) definen a las competencias como la capacidad que tiene un individuo para afrontar situaciones complejas en un contexto determinado; y para Tobón (2006) citado por Navarro et al. (2020 parr.15) las competencias emprendedoras permiten que los individuos desarrollen un proyecto empresarial o social que genere crecimiento económico y cohesión social, configurándose de esta manera como un proyecto social integrado.

Según Rodríguez y Orantes (2009 p.87) consideran que al hablar de competencia emprendedora hay que referirse a un ser humano que se adapta fácilmente a un mercado laboral flexible y cambiante, en el que se desenvuelve de manera creativa e innovadora.

Para la disciplina de enfermería no es nuevo enfrentarse a diferentes desafíos, en la historia de enfermería se encuentran presentes grandes trasformaciones sociales donde su participación ha sido fundamental para obtener resultados en salud de una sociedad, mostrando siempre una actitud de alto compromiso con la humanidad, por lo que adquirir una actitud dirigida a crear negocios en el cuidado de la vida y la salud y proyectar a la disciplina hacia una visión empresarial se convierte hoy, en un reto necesario en tiempos de crisis.

La actitud emprendedora representa la valoración personal, positiva o negativa, que los individuos sienten hacia el emprendimiento manifestando una fuerte orientación hacia la toma de decisiones y acciones. Así mismo se refiere a la autopercepción de la capacidad que desarrollan las personas para convertirse en empresarios (Vélez, Bustamante, Loor y Afcha 2020 p. 64).

\section{Metodología}

Se realizó una investigación documental de los programas de estudio de 5 universidades del país México a través de las plataformas digitales ISI Web of Knowledge, Google académicos, PubMed y SciELO. 
Posteriormente se definieron las variables teóricas con base al marco teórico que sustenta los diversas posturas y teórias del fenómeno a estudiar, se realizb la identificación de frecuencia, con lo que aparecieron las palabras claves continuamente, se definieron los constructos conceptuales de la investigación y finalmente se operacionalizaron las variables teóricas, realizando una primera versión del instrumento de recoleccion de información que constó de una ficha de identificación con 50 items.

Se realizó la primera validación con grupos de expertos, obteniendo retroalimentación y realizando las modificaciones sugeridas por el grupo de expertos, obteniendo una segunda versión, la cual se aplicó a un grupo piloto de 20 enfermeros egresados de una universidad local, obteniendo una segunda retroalimentación que implicó mejoras y finalmente desarrollar una tercera versión del instrumento que consta de ficha identificación y 20 reactivos.

Esta tercera versión se aplicó a una muestra seleccionada de 70 egresados de enfermeria de diferentes universidades del pais. Una vez creadas las observaciones se creo una base de datos en Excel, realizando el tratamiento estadístico con alfa de Crobanch con una confiabilidad de $>8$, con lo que se realiza la validación del mismo y permitió generar los resultados.

\section{Resultados}

Las universidades están invitadas a tener un papel proactivo para estimular el desarrollo económico, mediante su papel en la educación y en la absorción, adaptación y difusión de tecnología., son vistas como fuentes de habilidades técnicas industrialmente valiosas, facilitadoras del proceso de innovación y emprendimiento en el sistema de innovación de un país (Pedroza -Zapata et al. 2020 p. 102). Sin embargo, las universidades que ofrecen el plan de estudios de la profesión de enfermería no integran materias, cuyos contenidos ofrezcan conocimientos para la creación de negocios que permitan a los egresados ejercer la practica autónoma de enfermería.

Inclusión de materias con contenido académico para la creación de negocios en los planes de estudios donde cursaro n la licenciatura los profesionales de enfermería.

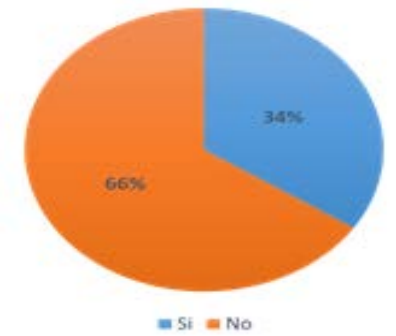

Figura 1. La figura demuestra el porcentaje de los profesionales de enfermería que durante su formación académica cursaron materias que incluyeran conocimientos para la creación de negocios, el 66\% responde que no cursaron una materia para la creación de negocios y un $34 \%$ responden que si cursaron una materia para la creación de negocios en enfermería. 
Inclusión de materias con contenido académico de mercadotecnia en servicios de salud en los planes de estudios donde cursaron la licenciatura los profesionales de enfermería

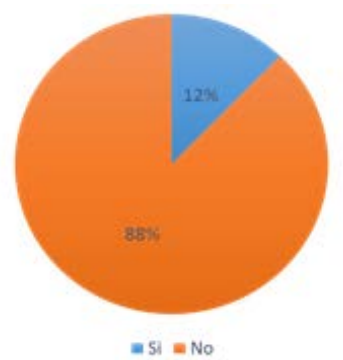

Figura 2. La figura 2 demuestra el porcentaje de los profesionales de enfermería que durante su formación académica cursaron la materia de mercadotecnia en servicios de salud el 88\% responden que no cursaron esta materia y el $12 \%$ responden que si cursaron la materia de mercadotecnia

Lugar de realización de prácticas profesionales de enfermería durante la formación académica de los profesionales de enfermería.

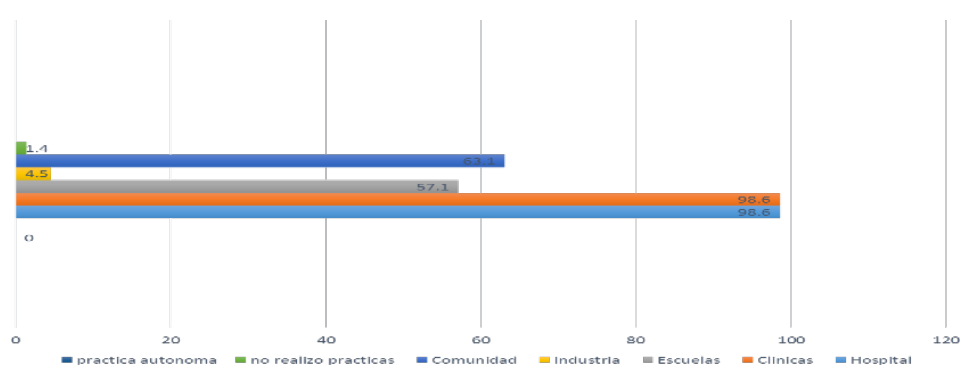

Figura 3. Los profesionales de enfermería entrevistados realizaron prácticas profesionales durante su formación académica en: consultorías de práctica autónoma de enfermería $0 \%$, profesionales que no realizaron prácticas en ningún lugar 1.4\%, el $63.1 \%$ realizaron prácticas en ámbito comunitario, el $4.5 \%$ en el ámbito industrial, el 57.7\% realizo prácticas en el ámbito educativo, el 98.6 \% realizó prácticas en el ámbito de clínicas de primer nivel de atención y 98.6 \% en ámbitos hospitalarios.

Influencia de los profesionales de enfermería para la creación de negocios de la disciplina durante la etapa de formación académica de los profesionales de enfermería.

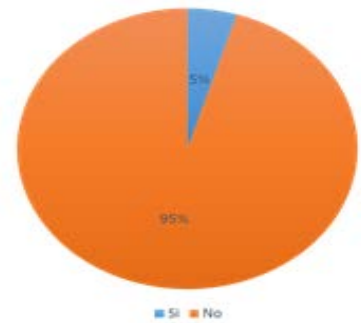

Figura 4. La figura demuestra que el 5\% de los profesionales de enfermería durante su formación académica recibieron influencia de profesionales de enfermería para la creación de negocios y el 95\% no recibieron influencia para la creación de negocios en salud durante su formación académica 


\section{Participación en proyectos de emprendimiento en salud de los profesionales de} enfermería durante el servicio social.

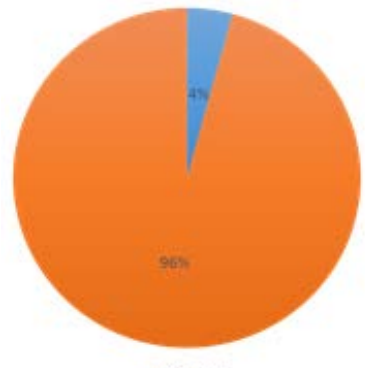

Figura 5. La figura 5 demuestra que los encuestados durante la realización del servicio social el $4 \%$ participó en proyectos de emprendimiento en salud y el 96\% no participó en proyectos de emprendimiento en salud

\section{Experiencia laboral de los maestros que impartieron cátedra durante la formación académica de los profesionales de enfermería.}

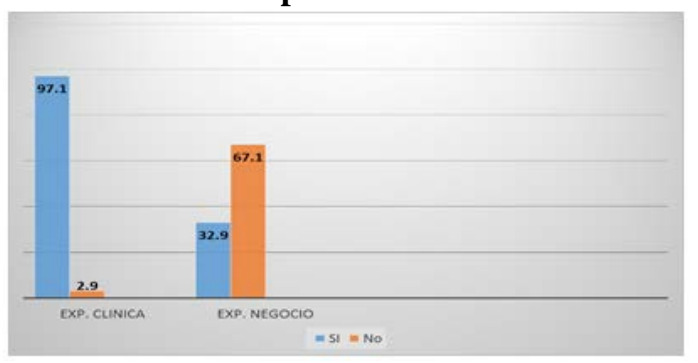

Figura 6. La figura 6 muestra la experiencia laboral de los maestros que impartieron cátedra a los encuestados, se observa que el 97.1\% poseían experiencia laboral clínica y el 2.9 no contaban con experiencia laboral clínica, el 32.9\% contaban con experiencia laboral en la creación de negocios y el 67.1\% no contaban con experiencia laboral en la creación de negocios

Observación de los profesionales de enfermería durante su formación académica de la participación de los catedráticos en proyectos de innovación en servicios de enfermería.

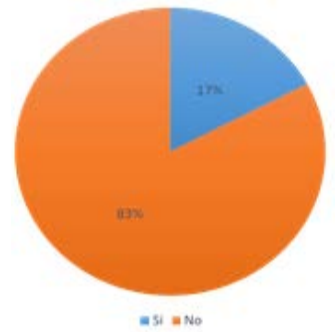

Figura 7. La figura 7 indica el porcentaje de los profesionales que durante su formación académica, observaron la participación de los catedráticos en proyectos de innovación en los servicios de enfermería, el 17 \% si observó la participación de los catedráticos y el 83\% no observo la participación de los catedráticos en proyectos de innovación de servicios de enfermería 


\section{Influencia ejercida de los catedráticos a los profesionales de enfermería durante su formación académica para ejercer la práctica autónoma de enfermería.}

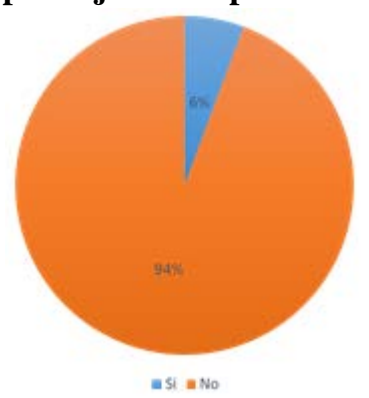

Figura 8. La figura 8 indica la influencia ejercida de los catedráticos que les impartieron clases a los profesionales de enfermería durante su formación académica, el 94\% de los entrevistados consideran que los catedráticos que no influyeron en ellos para ejercer una práctica autónoma de enfermería y el 6\% expresa que si influyeron en ellos para ejercer la practica autónoma de enfermería pero no la ejercen

\section{Discusión}

Rossetti, Aisa y Wright (2020 p.34) expresan que la vinculación Universidad-Empresa, requiere ser permanente para generar sinergia entre las demandas sociales que atienden la empresa y la formación de recursos humanos, futura fuerza laboral de las empresas con la intención de contribuir al desarrollo económico del país o bien de la región.

Según Bayas, García, Chaguay y Sánchez (2020 p.5) el emprendimiento ha retomado importancia para el desarrollo económico de un país, sin embargo las universidades que imparten la carrera de enfermería en México no lo han considerado al interior de los programas de estudios en la carrera de enfermería, ya que, como se observa los futuros profesionales no se tienen actividad durante su formación en conocimiento, ni en proyectos relacionados con el emprendimiento para el cuidado de la vida y la salud de las personas.

La transferencia de conocimientos y tecnologías entre el sector académico y la empresa pretende estimular la innovación, a manera de resolver problemáticas reales del sector productivo y de la sociedad, por lo que se considera importante la actualización oportuna de planes y programas de estudio que permita a los egresados responder a estas demandas sociales y empresariales actuales (Rossetti et al. 2020 p. 34).

La realización de las prácticas clínicas, así como el servicio social en enfermería son esenciales, pues significan la posibilidad de aplicar los conocimientos teóricos y adquirir habilidades indispensables para proporcionar cuidados al paciente, coadyuvando además a desarrollar una actitud crítica y humanista, fundamental para el futuro ejercicio profesional (Domez, Valencia y Contreras 2020 pp. 437-448). 
En esta población de estudio los encuestados indican que realizaron prácticas en diferentes ámbitos como son el ámbito hospitalario, en clínicas de primer y segundo nivel de atención, en comunidades, en ámbitos educativos y en un porcentaje mínimo en industrias, pero se observa que ninguno de los profesionales de enfermería entrevistado realizó prácticas profesionales con profesionales de enfermería que ejercieran la práctica autónoma del cuidado, por lo tanto no cuentan con un modelo de observación que les permita iniciar con la práctica autónoma del cuidado.

El crecimiento en el emprendimiento ha generado cambios en la dinámica global de desarrollo y creación de empresas y se ha resaltado la importancia de estimular a los jóvenes hacia la actividad emprendedora, pues son ellos los futuros empresarios y como tal está ligado el crecimiento futuro de las regiones (Mejía, Arias, \& Echeverri, 2017).

En el liderazgo docente para el emprendimiento según Guerrero et al. (2020 parr.2) expresan que el problema radica en que el docente actual desconoce el rol que debe asumir en estos contextos, bajo la figura de un docente emprendedor que a lo largo de su ejercicio docente logre incorporar la tecnología en procesos de innovación dentro del ámbito educativo, es por ello que se hace necesario que el docente en enfermería incursione en una pedagogía orientada hacia la resolución de conflictos, generación de competencias en liderazgo y emprendimiento.

Se observa que los académicos que impartieron clase a los entrevistados cuentan con experiencia clínica en primer y segundo nivel de atención, pero carecen de experiencia en proyectos de innovación o emprendimiento de la salud o de la disciplina de enfermería, lo que impide que puedan orientar a los estudiantes a adquirir una visión empresarial a futuro.

\section{Conclusión}

Los profesionales de enfermería al egresar de la formación académica no cuentan con conocimientos teórico prácticos para ejercer o liderar proyectos de emprendimiento para el cuidado de la vida y la salud de la población.

Los académicos y profesionales de enfermería que participan en el proceso de formación profesional, no cuentan con perfiles formativos que influyan en los alumnos para participar en proyectos de emprendimiento o visualizar el emprendimiento como una actividad remunerada en la profesión de enfermería.

Por lo tanto, se concluye que no existe liderazgo académico para el emprendimiento en enfermería, se sugiere a las universidades que ofertan la carrera de enfermería, impartir materias que permitan a los futuros profesionales la gestión de sus propios recursos económicos, así como actualizar los perfiles académicos de los catedráticos que imparten clases para impulsar la creación de 
consultorías en el cuidado de la vida y salud lideradas por profesionales de enfermería.

\section{References:}

1. Acosta Chávez, H. N., \& Leyva Solano, J. W. (2020). Autonomía profesional de la enfermera, estudio comparado: Perú (Trujillo) y México (Puebla). Repositorio instiucional de la Universidad Nacional de Trujillo. 1-87. Recuperado de http://dspace.unitru.edu.pe/handle/UNITRU/15956

2. Bayas, T. F., García, M. F. E., Chaguay, L. A. L., \& Sánchez, F. C. (2020). Tendencia de los estudiantes universitarios hacia el emprendimiento e innovación social. Journal of Science and Research: Revista Ciencia e Investigación, 5(1), 1-16. doi: org/10.5281/zenodo.3598484.

3. Benítez, J. (2020). La importancia de la investigación en Enfermería. Enfermería Investiga,5(1), 1-2. Recuperado de http://dx.doi.org/10.31243/enf.inv.v5i1.826.2020

4. Bermúdez, G. P. F. (2020). Enfermería y COVID-19: reconocimiento de la profesión en tiempos de adversidad. Revista Colombiana de Enfermería, 19(1), 1. Recuperado de https://doi.o rg/10.18270/rce.v19i1.2970

5. Colliere-MF.(2009). La importancia del cuidado de enfermería. Revista de enfermería del Instituto Mexicano del Seguro Social. 17 (2), 113-115

6. Domez, K. S. R., Valencia, L. P. P., \& Contreras, L. V. O. (2020). Estrés en estudiantes de enfermería que realizan prácticas clínicas en una institución universitaria de CartagenaColombia, 2019. Archivos de Medicina, 20(2). doi: https://doi.org/10.30554/archmed.20.2.3674.

7. Fernández, L. S., \& Rey, E. F. (2010). Competencias emprendedoras y desarrollo del espíritu empresarial en los centros educativos. Educación XX1, 13 (1), 15-38.

8. García, C. P., \& Gallegos-Torres, R. M. (2020). El papel del personal de enfermería en la educación para la salud. Revista Horizonte de Enfermería, 30(3), 271-285. doi: https://doi.org/10.7764/Horiz_Enferm.30.3.271-285.

9. Gatica, L., Cruz, E., Luis, K., García, P., Vargas, R., Hernández, J., \& Tepal. (2019). Liderazgo Docente en las Universidades Públicas de México. Revista Iberoamericana de Ciencias. 6(5). $1-12$. 
10. Guerrero, J. A. O., \& Conde, D. C. R. (2020). Papel del docente en el proceso de innovación en la pedagogía emprendedora. Gestión y Desarrollo Libre. 3(5).

11. Hernández, G. R. (2020). El cuidado de enfermería: una reflexión sobre el cuidado humanístico. Revista Horizonte de Enfermería. 23(2), 9-12.

12. Hernández, H. M. L., \& Romero, G. H. (2020). Liderazgo docente en la perspectiva de universitarios. Horizonte de la Ciencia. 10(18). Recuperado de https://doi.org/10.26490/uncp.horizonteciencia.2020.18.423

13. Rodríguez, F. M. M., \& Orantes, G. C. (2009). Aproximación al concepto de competencias emprendedoras: valor social e implicaciones educativas. Revista Iberoamericana sobre Calidad, Eficacia y Cambio en Educación. 7(3), 82-98. Recuperado de http://www.rinace.net/reice/numeros/arts/vol7num3/art6.pdf.

14. Mejía, J., Arias, C., \& Echeverri, L. (2017). El papel de la educación en creación. Revista CEA, 69-87.

15. Navarro, G. T., Bayona, J. A., \& Pacheco, C. A. (2020). Competencias emprendedoras y formación para el emprendimiento en instituciones de educación media. Revista Espacios, 41(11), 3-16.

16. Pedroza-Zapata, Á. R., \& Silva-Flores, M. L. (2020). Ecosistema Universitario de Ciencia, Tecnología, Innovación y Emprendimiento. Revista Internacional de Investigación en Educación. 12(25), 93-110.

17. Tobón, S. (2006). Aspectos básicos de la formación basada en competencias. Talca: Proyecto Mesesup, 1, 1-15.

18. Rossetti, G., Aisa, S., \& Wright, L. T. (2020). Análisis Comparativo entre las Metodologías de Vinculación Universidad-Empresa. Un Caso De Estudio. Revista Internacional de Ingeniería Industrial, (1), 34-45.

19. Santa Isaza, K. A. (2020). Modelos de atención de enfermería administrativos y asistenciales de asignación del personal de enfermería y su relación con la seguridad clínica y la satisfacción del paciente y del personal de enfermería. Repositorio Digital Institucional. 1-39. Recuperado de http:/hdl.handle.net/10946/4595.

20. Vélez, C. I., Bustamante, M. A., Loor, B. A., \& Afcha, S. M. (2020). La educación para el emprendimiento como predictor de una intención emprendedora de estudiantes universitarios. 
January 2021 edition Vol.17, No.3

Formación universitaria, 13(2), 63-72. Recuperado de http://dx.doi.org/10.4067/S0718-50062020000200063. 